\title{
Reabilitação sensório-motora de tetraplégicos através de estimulação elétrica neuromuscular*
}

\author{
The sensory-motor rehabilitation of tetraplegics via \\ neuromuscular electrical stimulation
}

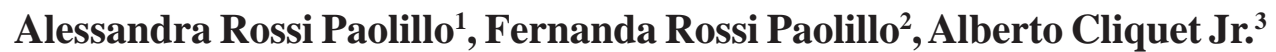

PAOLILLO, A. R.; PAOLILLO, F. R.; CLIQUET Jr., A. Reabilitação sensório-motora de tetraplégicos através de estimulação elétrica neuromuscular. Rev. Ter. Ocup. Univ. São Paulo, v. 17, n. 2, p. 72-79, maio/ ago., 2006.

RESUMO: O objetivo desta pesquisa consiste em verificar o ganho neurológico e a independência na execução de atividades funcionais em tetraplégicos durante o período de 6 meses de reabilitação com Estimulação Elétrica Neuro-Muscular (EENM). Foram realizados, em oito pacientes do sexo masculino, os Protocolos ASIA e FIM no período pré e pós tratamento que consistiu na Estimulação de membros inferiores e superiores, com realização de Atividades funcionais a partir da restauração de preensões. Os dados desta pesquisa foram analisados por meio de estudos de caso devido a variabilidade intersujeitos. Os tetraplégicos foram capazes de realizar todas as Atividades propostas, o que sem a EENM não seria possível. A recuperação sensório-motora foi maior nos membros superiores comparado aos membros inferiores. A maioria dos pacientes apresentou ganho neurológico com conseqüente diminuição do nível de lesão, bem como, aumento no índice de independência funcional. Portanto, estes efeitos terapêuticos da reabilitação através de EENM indicam plasticidade neural.

DESCRITORES: Pessoas portadoras de deficiência/reabilitação. Reabilitação. Terapia por estimulação elétrica. Plasticidade neuronal.

\footnotetext{
* Parte integrante da Dissertação de Mestrado de Alessandra Rossi Paolillo.

${ }^{1}$ Terapeuta Ocupacional e Mestre em Bioengenharia.

${ }^{2}$ Prof ${ }^{a}$. de Educação Física e Mestre em Bioengenharia.

${ }^{3}$ Engenheiro Eletricista, Ms. e Dr. em Bioengenharia, Livre Docente e Professor Titular da USP/UNICAMP.

Endereço para correspondência: Alessandra Rossi Paolillo - Rua Irmã Maria Saint Félix, 85. Jardim Cardinali — São Carlos, SP. CEP.: 13569-600.e-mail: arpaolillo@yahoo.com.br
} 


\section{INTRODUÇÃO}

A tecnologia assistida, como as neuropróteses por Estimulação Elétrica Neuro-Muscular (EENM) são utilizadas na reabilitação de portadores de deficiência neurológica, ampliando os recursos terapêuticos, especialmente no caso de portadores de lesão medular. A EENM consiste na ativação artificial dos músculos esqueléticos, o que possibilita sequiências de movimentos de membros superiores e inferiores, tais como, ficar em pé, caminhar, deslocar o membro superior no espaço para alcançar, pegar e soltar objetos (SEPÚLVEDA; CLIQUET Jr, 1998; CLIQUET; CASTRO, 2000).

Liberson, durante a década de 60 , foi o primeiro a utilizar a Estimulação Elétrica para o tratamento do pé em gota em portadores de hemiplegia, o eletrodo era posicionado no nervo fibular para evocar o reflexo de retirada. Long e Masciarelli, motivados por estes resultados, restauraram os movimentos da mão com a estimulação dos músculos extensores dos dedos, gerando atos simples de pegar e soltar objetos. Posteriormente, Kantrowitz utilizou a Estimulação Elétrica para restaurar a postura ereta de portadores de lesão medular, através da estimulação dos músculos quadríceps. Vodovinick, Kralj e Bajd iniciaram o desenvolvimento de estimuladores multicanais para possibilitar seqüências complexas de movimentos para dar passos (KRALJ; BAJD, 1989). Recentemente, os sistemas implantáveis também são incorporados (BHADRA et al., 2001).

As seqüelas após a lesão medular conduzem a diminuição da qualidade de vida, afetando em maior extensão os tetraplégicos, que apresentam maior insatisfação e sofrimento mental, devido a redução da funcionalidade física, acessibilidade, amizade, função social, ocupação, sexualidade e atividades de lazer, bem como o aumento da estigmatização, dependência e despesa financeira (MANNS; CHAD, 2001).

O termo tetraplegia é definido como a diminuição ou perda da função motora e/ou sensorial dos segmentos cervicais devido a lesão dos elementos neurais dentro do canal medular, resultando em redução da função dos membros superiores, tronco, membros inferiores e órgãos pélvicos (BARROS FILHO et al., 1994; BURNS; DITUNO, 2001).

De acordo com o nível de lesão, os tetraplégicos apresentam seqüelas típicas nos membros superiores, ou seja, os que sofreram lesão medular ao nível C4, geralmente apresentam controle escapular, mas não de todo braço. Uma lesão em C5, pode resultar em limitação do controle do ombro e flexão do cotovelo, embora estejam ausentes a extensão do cotovelo, punho e mão, bem como, ausência de sensibilidade abaixo do antebraço. Os portadores de lesão cervical C6, possuem mínima extensão de punho, acompanhada de sensibilidade parcial que estende-se distalmente para a mão. Em lesão ao nível C7-T1, os pacientes têm preservado algum controle dos dedos, acompanhado de sensibilidade parcial da mão (STEIN et al., 1992), o que resulta em impedimentos ou dificuldades na realização de atividades simples do cotidiano, como higiene pessoal, vestir-se, segurar objetos, entre outros.

Para restaurar e recuperar a função perdida dos membros superiores de tetraplégicos e melhorar sua independência e qualidade de vida, existem várias técnicas de tratamento, como as cirurgias de transferência de tendão, utilização de órteses, terapias convencionais e nãoconvencionais como a EENM (BHADRA et al., 2001).

A EENM, além de propiciar a restauração do movimento, quando aplicada durante período prolongado, aumenta a força e o volume do músculo, bem como reduz a espasticidade e rigidez (HANDA, 1997). Quanto a plasticidade muscular, foi constatado aumento no diâmetro das fibras musculares, aumento na proporção de fibras tipo I e da atividade enzimática oxidativa. Outros efeitos benéficos são a melhora da circulação sangüínea, da microcirculação e das trocas transcapilares. $\mathrm{O}$ aumento da perfusão de sangue no tecido muscular e na pele são importantes para a prevenção das úlceras de decúbito (DALY et al., 1996; KERN, 1997). As mudanças cardiovasculares abruptas observadas durante a postura sentada, ereta e durante o exercício com os membros superiores podem ser minimizadas com o uso da EENM nos membros inferiores, devido a ativação da bomba músculo-esquelética (HOPMAN et al., 1998; ELOKDA et al., 2000). O treinamento da marcha através de EENM em indivíduos lesados medulares possibilita a prevenção/ redução/reversão da osteoporose, por permitir a sustentação do peso corpóreo e atividade muscular, gerando aumento do estímulo mecânico para o remodelamento e crescimento ósseo, devido ao efeito piezoelétrico (CARVALHO et al., 2001). A postura bípede e o treinamento da marcha favorecem ainda o alívio da pressão na região glútea (MUCCIO et al., 1989), o trânsito intestinal, prevenção de infecção no trato urinário e contraturas articulares (THOUMIE et al., 1995).

$\mathrm{O}$ objetivo desta pesquisa foi verificar, durante o período de 6 meses de tratamento com EENM, se houve: (i) reabilitação sensório-motora com conseqüente ganho neurológico e (ii) maior independência na execução de atividades funcionais em tetraplégicos. 


\section{METODOLOGIA}

Esta pesquisa foi aprovada pelo Comitê de Ética da Universidade Estadual de Campinas (UNICAMP). Após o consentimento, participaram desta pesquisa oito portadores de tetraplegia do sexo masculino que fazem parte do Programa de EENM (Departamento de Ortopedia e Traumatologia, FCM/UNICAMP e Departamento de Engenharia Elétrica, USP/São Carlos). O perfil dos pacientes é apresentado na Tabela 1.

Tabela 1 - Perfil dos tetraplégicos participantes da pesquisa.

\begin{tabular}{|c|c|c|c|c|}
\hline $\begin{array}{l}\text { Pacientes } \\
\text { (anos) }\end{array}$ & Idade & $\begin{array}{c}\text { Nível de } \\
\text { lesão }\end{array}$ & Causa da lesão & $\begin{array}{l}\text { Tempo de } \\
\text { lesão (anos) }\end{array}$ \\
\hline 1 & 35 & $\mathrm{C} 7$ & $\begin{array}{l}\text { ferimento por } \\
\text { arma de fogo }\end{array}$ & 8 \\
\hline 2 & 31 & $\mathrm{C} 6-\mathrm{C} 7$ & $\begin{array}{l}\text { acidente } \\
\text { automobilístico }\end{array}$ & 12 \\
\hline 3 & 36 & C6 & $\begin{array}{l}\text { acidente } \\
\text { automobilístico }\end{array}$ & 2 \\
\hline 4 & 41 & C5-C6 & $\begin{array}{l}\text { acidente } \\
\text { automobilístico }\end{array}$ & 21 \\
\hline 5 & 26 & $\mathrm{C} 5$ & mergulho & 2 \\
\hline 6 & 37 & $\mathrm{C} 5$ & atropelamento & 7 \\
\hline 7 & 28 & $\mathrm{C} 4-\mathrm{C} 5$ & mergulho & 14 \\
\hline 8 & 24 & $\mathrm{C} 4$ & mergulho & 6 \\
\hline
\end{tabular}

A rotina de tratamento através de EENM é caracterizada por uma sessão semanal com duração aproximada de 60 minutos. Realiza-se a ativação dos músculos responsáveis pelos movimentos de flexão/extensão de punhos e dedos, além da estimulação dos músculos intrínsecos da mão durante 20 minutos. Posteriormente, realiza-se a estimulação dos músculos quadríceps (extensão dos joelhos) e dos nervos fibulares (dorsiflexão dos pés), respectivamente com duração de 20 e 15 minutos.

Para a implementação das sessões terapêuticas foram elaboradas e realizadas atividades funcionais. A elaboração das atividades foi determinada a partir do desejo dos pacientes. Desta maneira, para a realização de atividades lúdicas e funcionais foram restaurados os movimentos de preensão em pinça, disco, lateral e palmar, através da utilização de eletrodos de superfície e estimulador portátil com os seguintes parâmetros de estimulação: tipo de onda monofásica bipolar, ciclo de trabalho de $33 \%$, frequiência de $25 \mathrm{~Hz}$, duração de pulso de $300 \mu$ s e amplitude de 0 a $150 \mathrm{~V}$, ajustado para determinar a força de preensão. Os músculos estimulados foram: o primeiro dorsal interósseo e adutor do polegar, que possibilitaram as atividades lúdica (jogo de tabuleiro), de escrita, pintura e segurar uma moeda. Para o movimento de preensão em disco, foram estimulados os lumbricais e flexor do punho, que possibilitaram segurar uma tampa de frasco para mantimentos e um CD. Para preensão palmar, foram estimulados os lumbricais e abdutor do polegar, que possibilitaram segurar copos, telefone celular, secador e escova de cabelo. As atividades mais pedidas foram o jogo de tabuleiro e pintura em gesso (Figura 1).

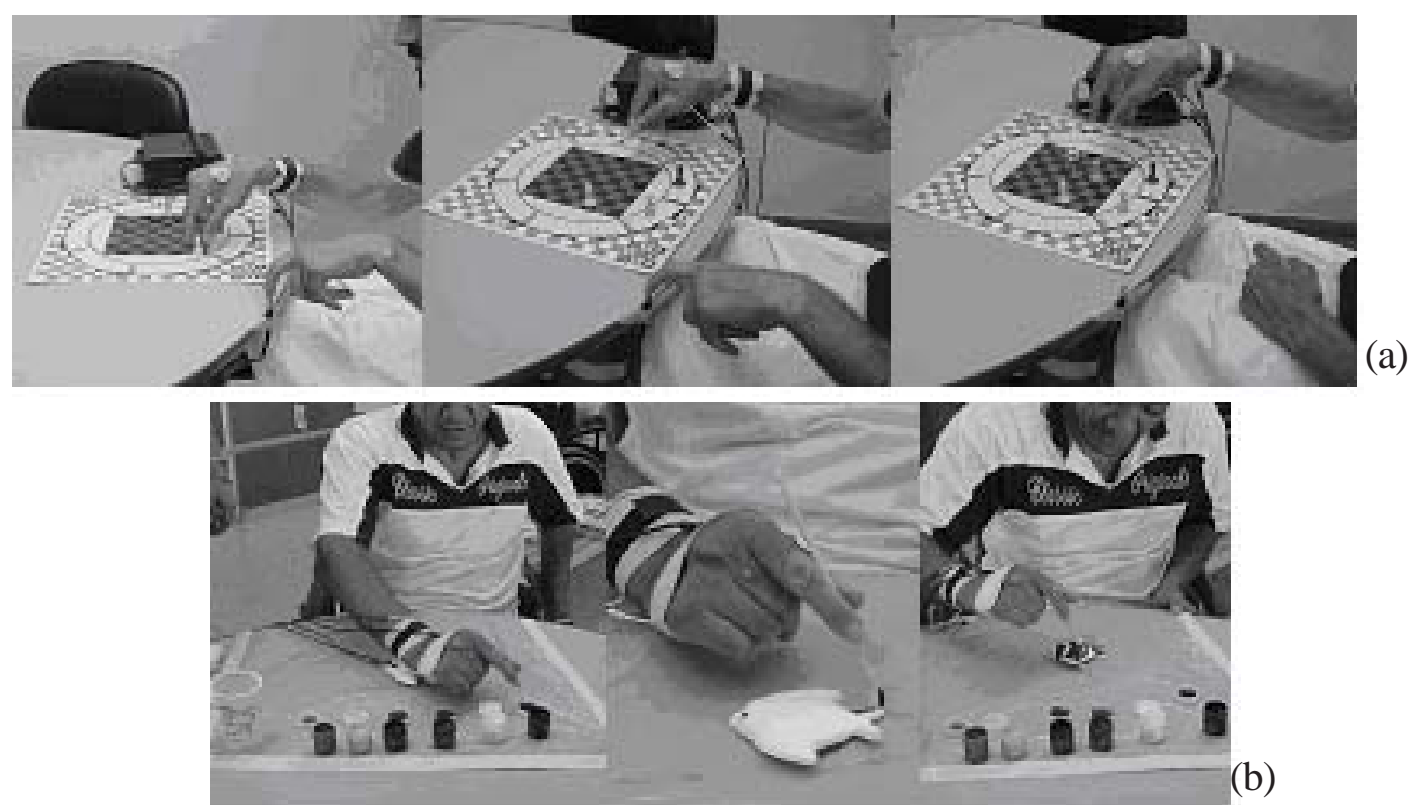

Figura 1 - Atividades Lúdicas desenvolvidas nas sessões terapêuticas: jogo de tabuleiro (a) e pintura em gesso (b). 
O interesse na plasticidade neural, durante a fase crônica da lesão medular e sua quantificação, surgiu a partir da melhora relatada pelos pacientes que fazem parte do Programa de EENM. Algumas de suas verbalizações indicam o retorno da sensibilidade e do movimento voluntário, por exemplo, que estavam sentindo novamente a temperatura da água durante o banho e que as mãos ou pés voltaram a mexer.

Foi definido por meio de revisão bibliográfica, o método mais apropriado para quantificar o possível retorno da sensibilidade e do movimento voluntário a baixo do nível de lesão, com conseqüente ganho neurológico (reabilitação sensório-motora).

Métodos quantitativos que requerem equipamentos sofisticados, como a eletromiografia e o potencial evocado, avaliam respectivamente o Sistema Neuro-Muscular e a integridade do Sistema Nervoso Central e Periférico (SMITH et al., 1999; CALANCIE et al., 2002).

Entretanto, uma avaliação qualitativa, fidedigna, rápida, de baixo custo e que não requer equipamentos sofisticados para avaliar a reabilitação sensório-motora é o Protocolo ASIA (escala de deficiência da American Spinal Injury Association), enquanto o Protocolo FIM (Functional Independence Measure), avalia o grau de independência funcional na execução das Atividades de Vida Diária (AVDs) descrevendo o impacto da lesão medular sobre o indivíduo, além de monitorar a evolução do tratamento. Estes Protocolos são recomendados pela American Spinal Injury Association (BARROS FILHO et al., 1994; BURNS; DITUNO, 2001).

O Protocolo ASIA consiste na realização do exame neurológico sensorial e motor, bilateramente, através dos dermátomos e miótomos. Foram examinados dois aspectos da sensibilidade, a dolorosa (com utilização de agulha) e o toque leve (com algodão). A sensibilidade foi classificada em ausente (0), comprometida (1) e normal (2). Os músculoschave examinados foram classificados de acordo com a contração obtida, variando da paralisia total (0), contração visível ou palpável (1), movimento ativo sem oposição da força de gravidade (2), movimento ativo contra a força da gravidade (3), movimento ativo contra alguma resistência (4), até movimento ativo contra grande resistência (5). Além da classificação do tipo de lesão completa (A) ou incompleta (B, C, D).

O Protocolo FIM avalia diversos aspectos das AVDs, como cuidados pessoais, controle de esfíncteres, mobilidade/transferência, locomoção, comunicação e cognição social, o que torna este método mais completo. Sua classificação ocorre em porcentagem, subdividido em níveis: com assistência (dependência completa ou modificada) e sem assistência (independência modificada ou completa), que permite a constatação das limitações e capacidades reais dos pacientes, que podem atingir o índice numérico máximo de 126.

Portanto, para determinar se houve reabilitação sensório-motora com conseqüente ganho neurológico e aumento do índice de independência funcional em um período de 6 meses de reabilitação com EENM, foram realizados os Protocolos ASIA e FIM no período pré e póstratamento.

\section{RESULTADOS}

O Paciente 1 apresentou aumento da força nos músculos flexores do cotovelo, com alteração na classificação de 4 para 5 do lado direito e de 3 para 4 do esquerdo, já o flexor profundo do terceiro dedo evoluiu de 3 para 4 do lado direito e de 0 para 1 do esquerdo. Nas áreas correspondentes ao dermátomo C8 (mão), a sensibilidade apresentou alteração na classificação do lado direito de 0 para 2 e do esquerdo de 0 para 1, em T1 (antebraços) e L1S3 (membros inferiores) de 0 para 1; além do aumento no índice de independência funcional (delta $=5)$.

O Paciente 2 obteve ganho de força nos extensores do punho, com conseqüente alteração na classificação de 4 para 5. A sensibilidade alterou-se de 1 para 2 nas áreas correspondentes aos dermátomos T1-T4 (antebraços/ região torácica); além do aumento no índice de independência funcional (delta $=9$ ).

O Paciente 3 apresentou aumento da força nos flexores do cotovelo do lado esquerdo, com alteração da classificação 4 para 5. Os extensores do punho obtiveram aumento de força com classificação de 4 para 5 em ambos os lados. O adutor do quinto dedo dos lados direito e esquerdo alteraram a classificação de 0 para 1 . A sensibilidade evoluiu na área correspondente ao dermátomo T3 (região torácica) do lado direito de 0 para 1 e do esquerdo de 0 para 2; além do aumento no índice de independência funcional $($ delta $=8)$.

O Paciente 4 apresentou aumento da força nos extensores do punho com classificação de 4 para 5 do lado esquerdo, igualmente aos flexores do cotovelo direito/ esquerdo e extensores do punho do lado direito. Os extensores do cotovelo alteraram a classificação de 3 para 4 do lado direito e de 1 para 3 do esquerdo. $O$ flexor profundo do terceiro dedo evoluiu de 0 para $1 \mathrm{em}$ ambos os lados. A sensibilidade aumentou nas áreas correspondentes aos dermátomos T1-T9 (antebraço/tórax/abdômen), com alteração da classificação de 0 para 1 ; além do aumento no 
PAOliLlo, A. R. et al. Reabilitação sensório-motora de tetraplégicos. Rev. Ter. Ocup. Univ. São Paulo, v. 17, n. 2, p. 72-79, maio/ago., 2006.

índice de independência funcional (delta =7).

O Paciente 5 apresentou aumento da força dos flexores do cotovelo com classificação de 4 para 5 e dos extensores do punho de 3 para 4 . A sensibilidade alterou-se na área dos dermátomos correspondentes a C5 (braço) do lado direito de 1 para 2, em C6 (antebraço/mão) de 1 para 2, em C8 (mão) de 0 para 2, em T2 (região axilar/tórax) de 1 para 2 e em T5 (tórax) de 0 para 1; além do aumento no índice de independência funcional (delta $=8$ ).

O Paciente 6 não apresentou aumento da força muscular nos membros superiores porém manteve as classificações obtidas. A sensibilidade alterou-se de 1 para 2 do lado direito nas áreas correspondentes aos dermátomos T11-S3 (região inferior do abdômen, membros inferiores, região glútea) e do lado esquerdo nas área de L2, L3, L5, S2 e S3 (coxa, joelho, pé, região poplítea e região glútea); além do aumento no índice de independência funcional $($ delta $=4)$.
O Paciente 7 apresentou alteração na classificação dos flexores do cotovelo do lado esquerdo de 0 para 1 e dos extensores do punho de ambos os lados de 0 para 1 . A sensibilidade alterou-se de 1 para 2 até os dermátomos correspondentes a $\mathrm{C} 4$ (ombro) e C5 (braço); além do aumento no índice de independência funcional (delta $=2$ ).

O Paciente 8 apresentou aumento da força no flexor do cotovelo do lado esquerdo com alteração na classificação de 4 para 5, para os flexores profundos do terceiro dedo de 0 para 1. A sensibilidade aumentou na área correspondente ao dermátomo C6 (tórax) e S2 (fossa poplítea), com alteração na classificação de 1 para 2 e de 0 para 1 respectivamente. $O$ paciente não apresentou aumento no índice de independência funcional.

A partir destes resultados determinou-se o nível de lesão neurológica mostrado na Tabela 2 , juntamente com o índice de independência funcional obtido por cada paciente.

Tabela 2 - Protocolos ASIA e FIM.

\begin{tabular}{|c|c|c|c|c|c|c|c|}
\hline \multirow[b]{2}{*}{ Pacientes } & \multirow[b]{2}{*}{ Avaliações } & \multicolumn{6}{|c|}{ ASIA: nível neurológico } \\
\hline & & Motor Direito & Motor Esquerdo & Sensorial Direito & Sensorial Esquerdo & Classificação & FIM* \\
\hline \multirow[t]{2}{*}{1} & Pré & $\mathrm{C} 7$ & $\mathrm{C} 4$ & $\mathrm{C} 7$ & $\mathrm{C} 4$ & A & 56 \\
\hline & Pós & $\mathrm{C} 8$ & $\mathrm{C} 5$ & $\mathrm{C} 7$ & C6 & A & 71 \\
\hline \multirow[t]{2}{*}{2} & Pré & $\mathrm{C} 7$ & $\mathrm{C} 7$ & T3 & $\mathrm{C} 7$ & B & 79 \\
\hline & Pós & $\mathrm{C} 7$ & $\mathrm{C} 7$ & $\mathrm{~T} 4$ & $\mathrm{C} 7$ & B & 88 \\
\hline \multirow[t]{2}{*}{3} & Pré & C7 & C7 & C7 & C7 & A & 61 \\
\hline & Pós & C7 & C7 & C7 & C7 & A & 69 \\
\hline \multirow[t]{2}{*}{4} & Pré & C6 & $\mathrm{C} 5$ & C6 & $\mathrm{C} 5$ & B & 64 \\
\hline & Pós & C6 & C6 & C7 & C6 & B & 71 \\
\hline \multirow[t]{2}{*}{5} & Pré & $\mathrm{C} 7$ & $\mathrm{C} 7$ & $\mathrm{C} 4$ & $\mathrm{C} 5$ & A & 66 \\
\hline & Pós & C7 & C7 & C6 & C5 & A & 74 \\
\hline \multirow[t]{2}{*}{6} & Pré & C7 & C7 & T10 & T10 & B & 92 \\
\hline & Pós & $\mathrm{C} 7$ & $\mathrm{C} 7$ & S3 & L1 & B & 96 \\
\hline \multirow[t]{2}{*}{7} & Pré & C5 & $\mathrm{C} 4$ & $\mathrm{C} 4$ & $\mathrm{C} 4$ & A & 53 \\
\hline & Pós & $\mathrm{C} 5$ & $\mathrm{C} 4$ & $\mathrm{C} 4$ & $\mathrm{C} 4$ & A & 55 \\
\hline \multirow[t]{2}{*}{8} & Pré & C5 & $\mathrm{C} 4$ & C6 & C6 & B & 77 \\
\hline & Pós & $\mathrm{C} 5$ & $\mathrm{C} 5$ & C6 & C7 & B & 77 \\
\hline
\end{tabular}

*Índice máximo: 126 
PAOlillo, A. R. et al. Reabilitação sensório-motora de tetraplégicos. Rev. Ter. Ocup. Univ. São Paulo, v. 17, n. 2, p. 72-79, maio/ago., 2006.

\section{DISCUSSÃO}

Estes resultados não podem ser comparados com os de outros estudos que utilizaram os protocolos ASIA e FIM, pois foram realizados com pacientes na fase aguda da lesão, no pós-cirúrgico e ainda na fase crônica, com tratamentos que visam a recuperação neurológica através da aplicação de novas drogas (TATOR, 1998; AKMAL et al., 2003). Entretanto, esta pesquisa foi realizada com pacientes na fase crônica da lesão durante o tratamento com EENM. Ainda, Programas de Reabilitação Sensório-Motora través de EENM são desenvolvidos por poucos grupos de pesquisa no mundo, como nos EUA, Canadá, Escócia, Japão e este no Brasil.

O protocolo ASIA mostrou que em um período de 6 meses de tratamento com EENM houve aumento de força muscular e sensibilidade principalmente nos membros superiores. Embora as respostas tenham sido individualizadas, a maioria dos pacientes apresentou ganho neurológico, com conseqüente diminuição do nível de lesão, bem como, aumento no índice de independência funcional.

Não foi constatada a piora do quadro clínico, uma vez que estes pacientes podem apresentar atrofia do sistema músculo-esquelético, redução da capacidade cárdio-vascular e respiratória, alterações metabólicas, hormonais e neuromusculares (EDGERTON et al., 2000), que juntamente com o estado sedentário podem ter como conseqüência a rápida instalação da fadiga, que reduz a independência nas atividades cotidianas e a qualidade de vida (DALLMEIJER et al., 1999).

A recuperação sensório-motora constatada foi maior nos membros superiores comparado aos membros inferiores na maioria dos pacientes. Portanto, a importância da EENM nos membros inferiores também consiste em benefícios hemodinâmicos devido a ativação da bomba muscular esquelética que favorece o retorno venoso e melhora a eficiência cardíaca de acordo com a Lei de Frank Starling (HOPMAN et al., 1998), bem como no aumento da força, massa e volume muscular (KERN, 1997), além de potencializar a plasticidade neural (CALANCIE et al., 1996; CALANCIE et al., 2002; CLIQUET Jr.; CASTRO, 2003).

A realização de atividades funcionais assistidas por EENM nos membros superiores são fundamentais, pois durante a execução das atividades propostas nas sessões terapêuticas, os pacientes demonstraram maior satisfação e motivação nas atividades lúdicas, que favorecem o aumento da auto-estima, iniciativa, criatividade, auto-expressão, organização de pensamento, orientação espaço-temporal, memória, bem como, a socialização. Outro aspecto relevante observado foi a coordenação motora fina e óculo-manual, gerada pelo ato de pegar as peças do jogo, através da preensão em pinça que foi acionada por botão, pelo membro contralateral, caracterizando uma atividade bimanual, que também ocorreu durante a realização da pintura em gesso, no qual houve o auxílio do outro membro para o posicionamento adequado do pincel, importante para o encontro dos membros superiores na linha média do corpo, favorecendo o desenvolvimento do esquema/imagem corporal e a integração sensório-motora. Estes aspectos são importantes, uma vez que encontram-se reduzidos ou ausentes na maioria dos portadores de lesão no sistema nervoso central (TROMBLY, 1989).

A reaprendizagem sensório-motora é obtida pela repetibilidade de movimentos dos membros superiores, ativados artificialmente, que geram input central e podem ser combinados com movimentos voluntários, conduzindo à reorganização cortical e aumento do ganho de função. Ainda, durante a realização das AVDs, utilizando Estimulação Elétrica e manipulação de objetos, há aumento do número de movimentos realizados com êxito e redução do tempo na execução das atividades (POPOVIC et al., 2002; POPOVIC, M.B. et al., 2002). Ainda, várias sessões diárias de prática, melhoram a velocidade e precisão nas tarefas motoras complexas, como as seqüências de movimentos rápidos (ROSSINI; PAURI, 2000).

Assim, o retorno da sensibilidade, do movimento voluntário e da marcha não-assistida em alguns pacientes paraplégicos e tetraplégicos que fazem/fizeram parte do Programa de EENM é explicado por recentes estudos neurofisiológicos realizados com potencial evocado e eletromiografia, baseados em movimentos sincrônicos e repetitivos que induzem à alterações nos circuitos interneurais da medula espinhal, por inputs aferentes (estímulo elétrico), possibilitando a reorganização dos reflexos, aumento da excitabilidade dos motoneurônios e a recuperação da função medular (PAOLILLO et al., 2002; CLIQUET Jr., 2002, 2003).

\section{CONCLUSÃO}

Portanto, com o processo de reabilitação através de EENM, a maioria dos tetraplégicos apresentou recuperação sensório-motora, com diminuição do nível de lesão neurológica e aumento no índice de independência funcional, além de realizarem todas as Atividades propostas o que sem a EENM não seria possível.

Agradecimentos: Os autores agradecem a Fundação de Pesquisa do Estado de São Paulo (FAPESP). - Processo n 96/12198-2. 
PAOLILLO, A. R.; PAOLILLO, F. R.; CLIQUET Jr., A. The sensory-motor rehabilitation of tetraplegics via neuromuscular electrical stimulation. Rev. Ter. Ocup. Univ. São Paulo, v. 17, n. 2, p. 72-79, maio/ ago., 2006.

\begin{abstract}
The objective of this research was to evaluate the independence to perform activities of daily living and neurological improvements due to a period of six months of Neuromuscular Electrical Stimulation (NMES). Eight tetraplegics participated in this study. The evaluations were accomplished with the ASIA and FIM Protocols. The treatment consisted in lower and upper limbs stimulation (prehension restauration). The results of this research was analised by case reports due the intersubject variability. The tetraplegics were able to perform all activities, wich without NMES was impossible to do. The sensory-motor recovery was higher in the upper limbs compared with lower limbs. The most patients showed neurological improvements and also in the index of functional independence due to the therapeutic effects of NMES.
\end{abstract}

KEY WORDS: Disabled persons/rehabilitation. Rehabilitation. Eletric stimulation therapy. Neural plasticity.

\title{
REFERÊNCIAS
}

AKMAL, M.; TRIVEDI, R.; SUTCLIFFE, J. Functional outcome in trauma patients with spinal injury. Spine, v. 28 , n. 2, p. 180$185,2003$.

BARROS FILHO, T. E. P.; de OLIVEIRA, R. P.; KALIL, E. M.; PRADA, F. S. Avaliação padronizada nos traumatismos raquimedulares. Rev. Bras. Ortop., v. 29, n. 3, p. 99-106, 1994.

BHADRA, N.; KILGORE, K. L.; PECKHAN, P. H. Implanted stimulators for restoration of function in spinal cord injury. Med. Eng. Phys., v. 23, p. 19-28, 2001.

BURNS, A. S.; DITUNO, J. F. Establishing prognosis and maximizing functional outcomes after spinal cord injury. Spine, v. 26, n. 245, p. 5137-5145, 2001.

CALANCIE, B.; LUTTON, S.; BROTON, J. G. Central nervous system plasticity after spinal cord injury in man: interlimb reflexes and the influence of cutaneous stimulation. Electroencephalogr. Clin. Neurophysiol., v. 101, p. 304-315, 1996.

CALANCIE, B.; MOLANO, M. R.; BROTON, J. G. Interlimb reflexes and synaptic plasticity become evident months after human spinal cord injury. Brain, v.125, p. 1150-1161, 2002.

CARVALHO, D. C. L.; CARVALHO, M. M.; CLIQUET Jr., A. Osteoporose por desuso: aplicação na reabilitação do lesado medular. Acta Ortop. Bras., v. 9, n. 3, p. 34-43, 2001.

CLIQUET Jr, A. A comprehensive sensorymotor rehabilitation programe for spinal cord injured patients using neuromuscular electrical stimulation. In: WORLD CONGRESS ON MEDICAL PHYSICS AND BIOMEDICAL ENGINEERING, Sydney, 2003. Anais... Sydney, Austrália, August, 2003. p. 24-29.

CLIQUET Jr., A. Conexão reativada. Rev. Pesq. FAPESP, n. 80, p. 48-51, 2002.
CLIQUET Jr., A.; CASTRO, M. C. F. An artificial grasping evaluation system for the paralysed hand. Med. Biol. Eng. Comput., v. 38 , n. 5, p. 275-281, 2000.

DALLMEIJER, A. J.; VAN DER WOUDE, L. H. V.; HOLLANDER, A. P.; VAN AS, H. H. J. Physical performance during rehabilitation in persons with spinal cord injuries. Med. Sci. Sports Exerc., v. 31, n. 9, p. 1330-1335, 1999.

DALY, J. J.; MARSOLAIS, E. B.; MENDELL, L. M.; RYMER, W. Z.; STEFANOVSKA, A.; WOLPAW, J. R.; KANTOR, C. Therapeutic neural effects of electrical stimulation. IEEE Trans. Rehabil. Eng., v. 4, n. 4, p. 218-230, 1996.

EDGERTON, V. R.; ROY, R. R.; HODGSON, J. A.; DAY, M. K.; WEISS, J.; HARKEMA, S. J.; DOBKIN, B.; GARFINKEL, A.; KONIGSBERG, E.; KOSLOVSKAYA, I. How the science and engineering of spaceflight contribute to understanding the plasticity of spinal cord injury. Acta Astronautica, v. 47, n. 1, p. 51$62,2000$.

ELOKDA, A. S.; NIELSEN, D. H.; SHIELDS, R. K. Effect of functional neuromuscular stimulation on postural related orthostatic stress in individuals with acute spinal cord injury. J. Rehabil. Res. Dev., v. 37, n. 5, p. 535-542, 2000.

HANDA, Y. Current topics in clinical functional electrical stimulation in Japan. J. Electromyogr. Kinesiol., v. 7, p. 269-274, 1997.

KRALJ, A. R.; BAJD, T. Functional electrical stimulation: standing and walking after spinal cord injury. Boca Raton, Fl.: CRC Press, 1989.

HOPMAN, M. T. E.; MONROE, M.; DUECK, C.; PHILLIPS, W. T.; SKINNER, J. S. Blood redistribution and circulatory responses to submaximal arm exercise in persons with spinal cord injury. Scand. J. Reabil. Med., v. 30, p. 167-174, 1998. 
PAOlillo, A. R. et al. Reabilitação sensório-motora de tetraplégicos. Rev. Ter. Ocup. Univ. São Paulo, v. 17, n. 2, p. 72-79, maio/ago., 2006.

KERN, H. Functional electrical stimulation in paraplegic spastic patients. Artif. Organs, v. 21, n. 3, p. 195-196, 1997.

MANNS, P. J.; CHAD, K. E. Components of quality of life for person with a quadriplegic and paraplegic spinal cord injury. Qual. Health Res., v. 11, n. 6, p. 795-811, 2001.

MUCCIO, P.; ANDREWS, B.; MARSOLAIS, E. B. Eletronic orthoses: technology, prototypes, and practices. J. Prosthet. Orthot., v. 1, n. 1, p. 3-17, 1989.

PAOLILlO, A. R.; PAOLILlO, F. R.; CLIQUET Jr., A. É possível voltar a andar após a lesão medular? In: CONGRESSO BRASILEIRO DE ENGENHARIA BIOMEDICA, 18., São José dos Campos, SP, setembro de 2002. Anais... São José dos Campos, 2002. p. 288.

POPOVIC, D. B.; POPOVIC, M. B.; SINKJAER, T. Neurorehabilitation of upper extremities in humans with sensorymotor impairement. Neuromodulation, v. 5, n. 1, p. 54-67, 2002.

POPOVIC, M. B.; POPOVIC, D. B.; TOMOVIC, R. Control of arm movement: reaching synergies for neuroprostheses with lifelike control. J. Automatic Control, v.12, p. 9-15, 2002.

ROSSINI, P. M.; PAURI, F. Neuromagnetic integrated methods tracking human brain mechanisms of sensorimotor areas "plastic" reorganization. Brain Res. Rev., v. 32, p. 131-154, 2000.
SEPÚLVEDA, F.; CLIQUET Jr., A. Gait restoration in a spinal cord injured subject via neuromuscular electrical stimulation controlled by an artificial neural network. Int. J. Artif. Organs, v. 21, n.1, p. 49-62, 1998

SMITH, H. C.; DAVEY, N. J.; SAVIC, G.; MASKILL, D. W.; ELLAWAY, P. H.; FRANKEL, H. L. Motor unit discharge characteristics during voluntary contraction in patients with incomplete spinal cord injury. Exp. Physiol., v. 84, p. 1151-1160, 1999.

STEIN, R. B.; PECKHAM, P. H.; POPOVIC, D. P. Neural prostheses: replacing motor function after disease or disability. New York: Oxford University Press, 1992. p. 163-187.

TATOR, C. H. Biology of neurological recovery and functional restoration after spinal cord injury. Neurosurgery, v. 42, p. 696708, 1998.

THOUMIE, P.; LE CLAIRE, G.; BEILLOT, J.; DASSONVILLE, J.; CHEVALIER, T.; PERROUIN-VERBE, B.; BEDOISEAU, M.; BUSNEL, M.; CORMERAIS, A.; COURTILLO, A. Restoration of functional gait in paraplegic patients with the RGO-II hybrid orthosis: physiological evaluation. Paraplegia, v. 33, p. 654-659, 1995.

TROMBLY, C. A. Terapia ocupacional para a disfunção física. São Paulo, SP: Santos, 1989.

Recebido para publicação: Maio/2006

Aceito para publicação: Maio/2006 